\title{
UWB Microwave Imaging for Inclusions Detection: Methodology for Comparing Artefact Removal Algorithms
}

\author{
James Puttock ${ }^{1}$, Behnaz Sohani ${ }^{1}$, Banafsheh Khalesi ${ }^{1}$, Gianluigi Tiberi ${ }^{1,2}$, Sandra \\ Dudley-McEvoy ${ }^{1}$ and Mohammad Ghavami ${ }^{1}$ \\ ${ }^{1}$ Department of Electrical and Electronic Engineering \\ London South Bank University, London UK \\ ${ }^{2}$ UBT - Umbria Bioengineering Technologies \\ Perugia, Italy
}

\begin{abstract}
An investigation is presented on Artefact Removal Methods for UltraWideband (UWB) Microwave Imaging. Simulations have been done representing UWB signals transmitted onto a cylindrical head-mimicking phantom containing an inclusion having dielectric properties imitating an haemorrhagic stroke. The ideal image is constructed by applying a Huygens' Principle based imaging algorithm to the difference between the electric field outside the cylinder with an inclusion and the electric field outside the same cylinder with no inclusion. Eight different artefact removal methods are then applied, with the inclusion positioned at $\pi$ and $-\frac{\pi}{4}$ radians, respectively. The ideal image is then used as a reference image to compare the artefact removal methods employing a novel Image Quality Index, calculated using a weighted combination of image quality metrics. The Summed Symmetric Differential method performed very well in our simulations.
\end{abstract}

Keywords: UWB Microwave Imaging, Image Quality Metric, Artefact Removal.

\section{Introduction}

In recent years, there has been considerable interest into research in the field of medical imaging. Current imaging techniques are advanced and can produce images of high clarity within a variety of tissue mediums. Medical professionals can draw on a range of technologies to assist with diagnosis depending on the suspected inclusion to be detected or the body part being imaged. Each technology has its own advantages and disadvantages. There are several techniques currently used for diagnosis purposes. Ultrasound scanners are cost-effective and successful in the medical diagnosis of areas of high contrast in soft tissues, hence their use in prenatal imaging and detection of various cancers. However, ultrasound is not a reliable technique for imaging air spaces, hard tissue such as bone/skull and providing definition in similar contrasting tissues. Computed Tomography (CT) is very good at imaging hard tissues but requires a substantial 
dose of ionising radiation. Magnetic Resonance Imaging (MRI) does not expose the patient to ionising radiation but does require them to stay still inside a claustrophobic space for a long time (up to hours). MRI and CT Scan devices are also expensive and not portable (owing to the pertinent dimensions of the devices), nor are they usable at rural medical centres, or carried by First Response Services (FRS) which are seen as essential for saving a person experiencing stroke. There is therefore an opportunity for research into a non-ionising, non-invasive, portable and cost-effective alternative.

Ultra-Wideband (UWB) technology has already proved successful in a number of areas related to health monitoring. The non-invasive nature of the signals and the success at detecting changes quickly in the wireless medium has proven effective in health monitoring through movement detection [1]. Recent research has also shown the successful detection of lesions which have different dielectric properties to the surrounding medium using UWB Microwave imaging [2]. This holds the potential for detections of cancer and stroke [3-5].

Current UWB microwave imaging methods rely on algorithms to process the electric field measured at various points around the perimeter of an object. With all the algorithms used there is the risk of inaccurate results due to reflections of the transmitting signal and unwanted reflections of signals from the surrounding tissues. These unwanted signals are known as 'artefacts' [6]. For the successful application of any imaging apparatus, a reliable artefact-removal algorithm is necessary.

This study will explore a methodology for analysing and comparing a variety of methods for removing artefacts, using several imaging and signal processing metrics to provide a weighted Image Quality Index.

\section{Theoretical Framework}

Previous study focussing on breast cancer imaging [7] has identified that microwaves respond differently if they hit tissues which have different dielectric properties. By using UWB across microwave frequencies, it is possible to produce images with enough resolution to show inclusions. The same principle has been used to determine the contrast between blood and brain matter to identify stroke in head-mimicking phantoms [8].

This study uses a technique explained in [9] which uses Huygens' Principle (HP) to forward propagate the waves [10]. This avoids having to solve complex inverse problems. A simulated waveform is constructed using the principles laid out by Parrikar et al [11], which is transmitted from a line source external to the cylinder and received at a point on the radius, external or at the edge of the cylinder. The electric field $E$ can be calculated by summing the known Electric Field $E_{\text {known }}$ at $\mathrm{N}_{\mathrm{PT}}$ points $n p$ on the perimeter using equation (1). HP indicates the Huygens' Principle method used and $G$ refers to the use of Green's function.

$$
E_{H P}\left(r, \phi ; \theta ; t x_{m} ; f\right)=\Delta_{s} \sum_{n p=1}^{N_{P T}} E_{n p, t x_{m}}^{\text {known }} G\left(k_{1}\left|\vec{r}_{n p}-\vec{r}\right|\right)
$$


where $(r, \phi, \theta) \equiv \vec{r}$ is the observation point, $k_{1}$ represents the wave number of the media constituting the cylinder, $\Delta_{s}$ is the spatial sampling, and $t x_{m}$ is the transmitting line source operating at frequency $f$.

An image is obtained by summing the solutions and mapping the intensity values. With $N_{f}$ frequencies $f_{i}$, the intensity of the final image $I$ can be obtained using equation (2).

$$
I\left(r, \phi, \theta ; t x_{m}\right)=\frac{1}{B} \sum_{i=1}^{N_{f}} \Delta_{f}\left|E_{H P}\left(r, \theta, \phi ; t x_{m} ; f\right)\right|^{2}
$$

where $\Delta_{f}$ and $B$ are the frequency sampling and Bandwidth, respectively.

By subtracting, before applying equation (1), the electric field with no inclusion from the electric field with an inclusion to get the difference in electric fields $E^{\text {diff }}$ :

$$
E_{n p, t x_{\mathrm{m}}}^{\text {diff }}=E_{n p, t x_{\mathrm{m}}}^{\text {known(With Inclusion) }}-E_{n p, t x_{\mathrm{m}}}^{\text {known(No Inclusion) }}
$$

the image obtained through equation (2) will show the inclusion.

For the purposes of real scenario medical imaging, equation (3) cannot be used. In [6], it has been shown that this problem can be solved by producing a matrix of average values of the electric field obtained when the inclusion is present. Measurements are taken from multiple transmission sources and a mean value generated, which is subtracted from the single transmitter data. This is explained mathematically in equation (4).

$$
E_{H P}\left(r, \phi, \theta ; t x_{m} ; f\right)=\Delta_{s} \sum_{n p=1}^{N_{P T}}\left(E_{n p, t x_{m}}^{\text {known }}-\operatorname{avg}_{M}\left\{E_{n p, t x_{m}}^{\text {known }}\right\}\right) \times \mathrm{G}\left(k_{1}\left|\vec{r}_{n p}-\vec{r}\right|\right)
$$

The Average subtraction method represents just one method of obtaining an image of the inclusion. Several methods have been proposed which replace the average matrix with an alternative, such as the measurements from a neighbouring transmitter in the case of Rotation Subtraction [6,12], or by using a differential method of obtaining the resulting image, as is the case in [2,13-14]. Variations on these techniques will be simulated and compared in this study.

\section{$3 \quad$ Methodology}

\subsection{Ideal Image Construction}

For the simulations, an external cylinder was simulated with radius $7 \mathrm{~cm}$, relative dielectric constant $\epsilon r=10$ and conductivity $\sigma=0.2 \mathrm{~S} / \mathrm{m}$. An internal cylindrical inclusion was constructed with radius $0.5 \mathrm{~cm}$, relative dielectric constant $\epsilon r=60$, conductivity $\sigma=2 \mathrm{~S} / \mathrm{m}$ and located $2 \mathrm{~cm}$ from the centre of the external cylinder with an eccentricity angle of $-\frac{\pi}{4}$ radians. A simulation was run to construct the electric field at the perimeter of the external cylinder. This field was simulated using MATLAB and generated a value for the electric field at 80 points around the circumference for 1101 discrete frequencies between 1 and $6.5 \mathrm{GHz}$. The normalised microwave image of such cylinder with an inclusion can be seen in Fig. 1 (a). $x$ and $y$ values are in metres. 
As can be seen in Fig. 1 (a), the electric field displays the reflection of the transmitter signal on the right of the cylinder. The transmitting signal has been set as a line source external to the cylinder at $x=-0.2, y=0$. No evidence of the inclusion can be seen in this image. This is because the reflected transmitter signal is greater by a significant order of magnitude than the reflections from the inclusion.

Fig 1(b) shows instead the image of the cylinder without the inclusion. Fig. 1 (a) looks very similar to Fig. 1 (b). To detect the inclusion, equation (3) should be used before performing imaging: such an image is given in Fig. 1 (c) and is used as an 'Ideal Image' for reference and comparisons.

This process is repeated with an inclusion at an eccentricity angle of $\pi$ radians and the images used as reference images for comparing subsequent experiments using different pre-processing algorithms.

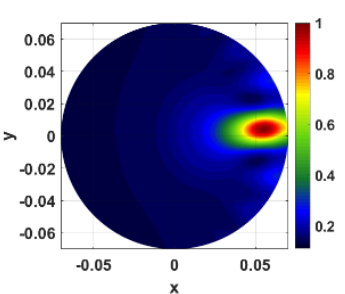

(a)

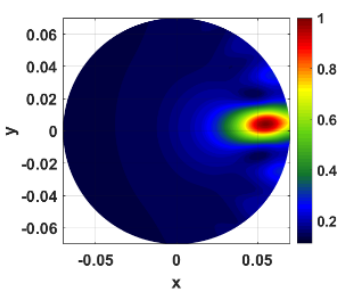

(b)

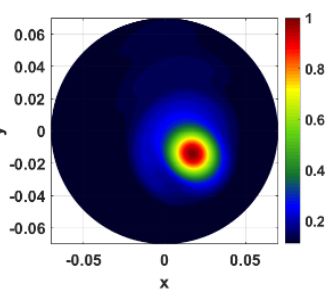

(c)

Fig. 1. (a) Image of cylinder with an inclusion, and (b) without inclusion. (c) Ideal image (constructed after employing equation (3)).

\subsection{Artefact Removal Methods}

This study involves a comparison of various artefact removal algorithms operating in the frequency domain.

Average Subtraction (AS). The average subtraction method works by taking the results from multi-transmitting sources ( 3 in this case) positioned slightly apart from each other $\left(4.5^{\circ}\right.$ in this case $)$ on the perimeter of the cylinder, with the first transmitter $x_{1}$ positioned at $x=-0.07, y=0$. When tabulating the (known) Electric Field at $n p$ points on the perimeter, the data from transmission point $x_{1}$ are placed into matrix $A_{1}$ which has dimensions made up by the number of frequencies $\times$ number of observation points. For this series of experiments, 1101 discrete frequencies $f$ are used at 80 observation points, here denoted with $\phi$, giving matrix $A_{1}$ in equation (5).

$$
A_{1}=\left[\begin{array}{ccc}
f_{1} \phi_{1} & \cdots & f_{1} \phi_{80} \\
\vdots & \ddots & \vdots \\
f_{1101} \phi_{1} & \cdots & f_{1101} \phi_{80}
\end{array}\right]
$$

The results from transmission points $x_{2}$ and $x_{3}$ are placed into matrices $A_{2}$ and $A_{3}$ respectively in a similar fashion. Next, the mean of each point is calculated, i.e. $A_{\text {avg }}$. 
The resulting average matrix $A_{\text {avg }}$ is then subtracted from the matrix of the first transmitter $A_{1}$ before performing imaging:

$$
E_{A S}=A_{1}-A_{\text {avg }}
$$

Rotation Subtraction (RS). The simulation is set up to replicate a signal from 2 transmitters, placed $4.5^{\circ}$ apart on the perimeter of the cylinder. The receivers, frequencies and other input parameters remain the same as for the previous experiments. The image is then constructed using matrix $E_{R S}$, given as the following:

$$
E_{R S}=A_{1}-A_{2}
$$

where $A_{1}$ and $A_{2}$ are the results matrix from transmitter 1 and 2 , respectively.

Differential Neighbouring Receiver Type (DNR). This method is adapted from Klemm's Differential method [2]. Instead of using the raw results to build a matrix from, the input matrix is instead built using the difference in value between neighbouring receivers. The input matrix $\mathrm{S}$ is thus built using the following calculation.

$$
\begin{gathered}
S(f, \phi ; n)=A(f, \phi: n)-A(f, \phi-1 ; n) \\
\text { for } \phi=1 \text { to } \frac{N_{a}}{2} \text { with } \phi-1=\mathrm{N}_{\mathrm{a}} \text { for } \phi=1 \\
S(f, \phi ; n)=A(f, \phi, n)-A(f, \phi+1 ; n) \\
\text { for } \phi=\frac{N_{a}}{2}+1 \text { to } N_{a} \text { with } \phi+1=1 \text { for } \phi=N_{a}
\end{gathered}
$$

where $N_{a}$ is the number of receiving antennas, $n$ is the transmitter index and $A$ is the original results matrix. This results in a Differential (Neighbouring Receiver Type) matrix $S$. To such a matrix is then applied the Average Subtraction or Rotation Subtraction methods.

Differential Symmetric Receiver Type (DSR). By exploiting the (eventual) object symmetry, it may also be possible to construct a differential matrix using the difference between the receivers placed symmetrically opposite. This is adapted from a method used by Mustafa et al. in [11]. The differential matrix $\mathrm{S}$ is built by subtracting each receiver value from its symmetrically opposite receiver as in equation (10).

$$
S(f, \phi ; n)=A(f, \phi ; n)-A\left(f, N_{a}+2-\phi ; n\right)
$$

for $\phi=1$ to $N_{a}$ with $N_{a}+1=\frac{N_{a}}{2}+1$ for $\phi=1$ and

$$
\frac{N_{a}}{2}+1=1 \text { for } \phi=\frac{N_{a}}{2}+1 \text {. }
$$


This results in a Differential (Symmetric Receiver Type) matrix $S$. To such a matrix is then applied the Average Subtraction or Rotation Subtraction methods.

Summed Symmetric Differential (SSD). The Symmetric Differential method above relies on the natural symmetry of some objects, such as the brain, across the left and right halves. However, there is a risk with the symmetric method of mirrored artefacts appearing in the images. The ellipsoid shape of the skull and brain have a distinct leftright line of symmetry. The front-back sections of the brain also contain similar densities of tissue. Whilst not completely symmetrical, the similarity in shape and density could be utilised to provide an artefact removal method by summing a differential matrix formed from the left-right differential and a second matrix formed from a frontback differential. This should provide a more intense peak at the area of inclusion and mirrored artefacts should have a reduced intensity. As before, a differential matrix $S$ is constructed as in equation (10). A second matrix $\mathrm{R}$ is constructed across the front-back receivers as follows.

$$
\begin{gathered}
R(f, \phi ; n)=A(f, \phi ; n)-A\left(f, \frac{N_{a}}{2}+2-\phi ; n\right) \\
\text { for } \phi=1 \text { to } \frac{N_{a}}{2}+1 \\
\text { with } \frac{N_{a}}{2}+2-\phi=\frac{3 N_{a}}{4}+1 \text { for } \phi=\frac{N_{a}}{4}+1 \\
R(f, \phi ; n)=A(f, \phi ; n)-A\left(f, \frac{3 N_{a}}{2}+2-\phi ; n\right) \\
\text { for } \phi=\frac{\mathrm{N}_{\mathrm{a}}}{2}+2 \text { to } N_{a} \\
\text { with } \frac{3 N_{a}}{2}+2-\phi=\frac{N_{a}}{4}+1 \text { for } \phi=\frac{3 N_{a}}{4}+1
\end{gathered}
$$

where $N_{a}$ is the number of receiving antennas, $n$ is the transmitter index and $A$ is the original results matrix. The combined matrix $C$ is then constructed by summing matrices $S$ and $R$.

$$
C(i, j ; n)=S(i, j ; n)+R(i, j ; n)
$$

This results in a Differential (Summed Symmetric Receiver Type) matrix. To such a matrix is then applied the Average Subtraction or Rotation Subtraction methods.

\subsection{Comparison Methods}

To compare the proposed artefact removal methods, it is necessary to construct a quantifiable measurement system that can be used to compare images. It is often difficult to construct a useful quantifiable number to measure an image. Whilst humans are good at recognising patterns and contrasts in an image, a machine must be taught each 
process, and this uses considerable computing power. For this experiment, basic comparison metrics have been calculated to compare the results. These are explained in greater detail below. Some of the below metrics rely on a reference image. For the purposes of this experiment, an 'Ideal Image' has been used as shown above.

Error Image. An Error image $M_{E r r}$ is constructed by subtracting the ideal image $X_{\text {Ideal }}$, from the image to be tested $Y_{\text {Test }}$.

$$
M_{E r r}=Y_{\text {test }}-X_{\text {Ideal }}
$$

Mean Square Error (MSE). The mean square error is the mean of all the squared values of the errors in the error matrix. Squaring the values means that any negative values become positive, so the absolute value is important. The average gives a single value which is an indication of the error across the whole matrix.

$$
\text { MSE }=\frac{1}{N} \sum_{i=1}^{N} M_{E r r_{i}}^{2}
$$

where $N$ is the number of elements in the Error matrix $M_{E r r}$. The mean square error can be calculated using MATLAB's imaging toolbox and the command immse [15].

Polyshape Construction. To evaluate the shape of the inclusion, we set 0.75 as a threshold on the normalised image, assigning every value above 0.75 to 1 , and all others to 0 . The resulting shape can then be obtained using MATLAB's polyboundary and polyshape functions [15].

Area Difference (ArD). This metric is related to the comparison between the size of the target area for an 'Ideal' image and the size of the target area in the test image.

Centroid Difference (CD). To test the accuracy of the image at locating an inclusion, the above Polyshape method was combined with MATLAB's centroid function. Comparison of accuracy can be made by assessing the Euclidean difference between the centroid of an ideal image polyshape and the test image. This will assess how accurate the location of the inclusion in the test image is. This is done using the MATLAB pdist function [15].

Signal-to-Noise Ratio (SNR). The Signal-to-Noise Ratio (SNR) is a useful metric in determining how clear any detected inclusion is by providing an assessment of the ratio between the background noise and the desired signal. To calculate the Signal-to-Noise Ratio (SNR) in dB, the above threshold is used to calculate the Polyshape to determine the target and background areas. SNR calculations are performed based on this result. This method is described in [12] and can be calculated using equation (16). 


$$
\mathrm{SNR}=10 \log _{10}\left(\frac{Q_{t}-Q_{b}}{D_{b}}\right) \mathrm{dB}
$$

where $Q_{t}$ and $Q_{b}$ are the mean values of the detected target and background regions, respectively, and $D_{b}$ is the standard deviation of the background.

Structural Similarity Index Metric (SSIM). The SSIM is an image quality metric which gives a value between 0 and 1 which indicates the similarity between two images (with 1 meaning the images are identical) [16]. This is calculated using the following equation.

$$
\operatorname{SSIM}=\frac{(2 \times \bar{x} \times \bar{y}+C 1)\left(2 \times \sigma_{x y}+C 2\right)}{\left(\sigma_{x}^{2}+\sigma_{y}^{2}+C 2\right)\left(\bar{x}^{2}+\bar{y}^{2}+C 1\right)}
$$

where $x$ is the reference image, $y$ is the test image, $\bar{x}$ and $\bar{y}$ represent the corresponding mean, $\sigma_{x}$ and $\sigma_{y}$ represent the corresponding variance, $\sigma_{x y}$ is the covariance of the reference and test image and $C 1$ and $C 2$ are small constants. MATLAB can calculate the SSIM based on two input images using the ssim function [15]. This will output both a value and a monochrome mapping which is a useful visual assessment of the quality of the image. An example is shown in Fig. 2(e).

Average Difference (AvD). The average difference is a measure of the mean difference in value between the Ideal Image and the test image. It is calculated by summing the elements of the Error Matrix and dividing by the number of elements.

Image Quality Index (IQI). The above metrics provide several ways of quantifying the precision, accuracy, and quality of the images to be constructed. Whilst these metrics are useful, to aid in comparing the methods an overall quality index will be calculated. This will be constructed by giving each of the metrics a score between 1 and 0 (with 1 being a perfect image or match with the Ideal image). For many of these values, as they are already being based on normalised results which will be between 0 and 1 , it is relatively simple to produce an appropriate score. For SNR, instead a comparison is made with the Ideal Image. The value will approach 1 as it approaches the SNR value of the Ideal image. The full metric Indexes $I$ are shown in equations (18-23) with $R$ representing the test result value.

$$
\begin{aligned}
& I_{M S E}=1-\mathrm{MSE}_{R} \\
& I_{S N R}=\frac{\mathrm{SNR}_{R}}{\mathrm{SNR}_{\text {Ideal }}} \\
& I_{S S I M}=\mathrm{SSIM}_{R} \\
& I_{A v D}=1-\mathrm{AvD}_{\mathrm{R}} \\
& I_{C D}=1-\frac{\mathrm{CD}_{R}}{\text { External Cylinder Radius }}
\end{aligned}
$$




$$
I_{A r D}=1-\frac{\operatorname{ArD}_{R}}{\text { External Cylinder Area }}
$$

The overall Image Quality Index is then calculated by taking a weighted average of all the indexes. The most useful metrics for our study will be ones that measure the accuracy, precision, and quality of the image. Therefore, Area Difference Index $I_{A r D}$, Centroid Difference $I_{C D}$ and Signal-to-Noise Ratio $I_{S N R}$ are each given a weighting of 0.25 . The SSIM value $I_{S S I M}$ provides a very useful measure of the contrast differences between the test image and ideal image. This has been given a weighting of 0.15 . The Average Difference $I_{A v D}$ and Mean Square Error $I_{M S E}$ provide useful additional information but are considered a less reliable assessment of quality as error value can easily be influenced by the power of received signals without necessarily affecting the ability to detect inclusions. These have therefore been given a weighting of 0.05 each. These values have been chosen arbitrarily based on the measurement requirements of this project.

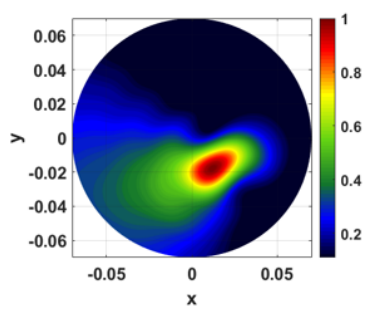

(a)

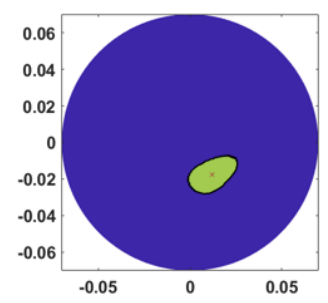

(d)

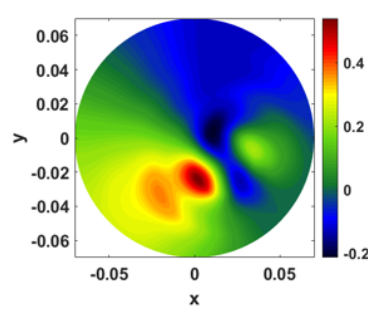

(b)

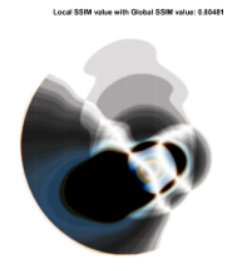

(e)

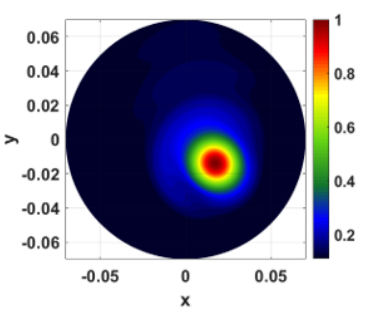

(c)

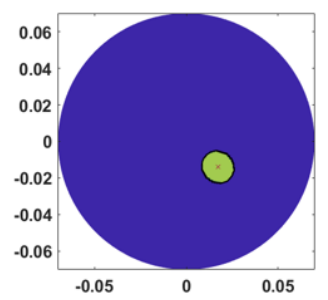

(f)

Fig. 2. Average Subtraction Results Images (a) Results Image, (b) Error Image, (c) Ideal Image, (d) Results Polyshape, (e) SSIM Image and (f) Ideal Polyshape.

\section{$4 \quad$ Results}

The simulation was run using each of the 5 Artefact removal methods, with the differential methods being calculated using an Average Subtraction or Rotation subtraction sub-method on the differential matrix. The results are presented for an inclusion at $\pi$ radians (Table 1) and for an inclusion at $-\frac{\pi}{4}$ radians (Table 2). The experiment names and metrics are expressed in abbreviated form. The Ideal SNR value was calculated following the construction of the Ideal Images and had a value of approximately 7.4 
dB. The results images for an inclusion at $-\frac{\pi}{4}$ radians are shown in Fig. 3. A subset of the results images for Average Subtraction (AS) methods on an inclusion at $\pi$ radians are shown in Fig. 4.

Table 1. Artefact Removal Comparison with Inclusion at $\pi$ radians.

\begin{tabular}{lllllllll}
\hline $\begin{array}{l}\text { Experi- } \\
\text { ment }\end{array}$ & $\begin{array}{l}\text { Subtrac- } \\
\text { tion } \\
\text { Method }\end{array}$ & MSE & $\begin{array}{l}\text { SNR } \\
{[\mathrm{dB}]}\end{array}$ & SSIM & AvD & $\begin{array}{l}\text { CD } \\
{[\mathrm{mm}]}\end{array}$ & ArD & IQI \\
\hline AS & N/A & 0.09729 & 5.36519 & 0.70400 & 0.25517 & 1.21322 & 0.0018 & 0.90422 \\
RS & N/A & 0.09937 & 5.44750 & 0.70809 & 0.25663 & 1.40726 & 0.0005 & 0.90775 \\
DNR & Average & 0.09814 & 6.06145 & 0.71389 & 0.25937 & 13.06835 & 0.0233 & 0.88642 \\
DNR & Rotation & 0.10098 & 6.04735 & 0.71476 & 0.26439 & 11.70547 & 0.0179 & 0.89186 \\
DSR & Average & 0.09974 & 5.40451 & 0.69871 & 0.25736 & 2.43316 & 0.0008 & 0.90070 \\
DSR & Rotation & 0.10008 & 5.44746 & 0.69865 & 0.25737 & 1.15772 & 0.0000 & 0.90726 \\
SSR & Average & 0.09462 & 6.03662 & 0.73537 & 0.23888 & 3.33967 & 0.0061 & 0.92886 \\
SSR & Rotation & 0.09523 & 6.06927 & 0.73697 & 0.23870 & 2.94882 & 0.0072 & 0.93161 \\
\hline
\end{tabular}

Table 2. Artefact Removal Comparison with Inclusion at $-\frac{\pi}{4}$ radians.

\begin{tabular}{lllllllll}
\hline $\begin{array}{l}\text { Experi- } \\
\text { ment }\end{array}$ & $\begin{array}{l}\text { Subtrac- } \\
\text { tion } \\
\text { Method }\end{array}$ & MSE & $\begin{array}{l}\text { SNR } \\
{[\mathrm{dB}]}\end{array}$ & SSIM & AvD & $\begin{array}{l}\text { CD } \\
{[\mathrm{mm}]}\end{array}$ & ArD & IQI \\
\hline AS & N/A & 0.02934 & 5.66908 & 0.80481 & 0.13335 & 6.14234 & 0.0143 & 0.87828 \\
RS & N/A & 0.03214 & 5.59032 & 0.80104 & 0.13886 & 7.21017 & 0.0164 & 0.86969 \\
DNR & Average & 0.06838 & 3.55544 & 0.73731 & 0.20546 & 9.19064 & 0.0159 & 0.78002 \\
DNR & Rotation & 0.05846 & 3.94577 & 0.74968 & 0.19013 & 11.2011 & 0.0000 & 0.79266 \\
DSR & Average & 0.06179 & 4.60431 & 0.75460 & 0.19962 & 14.3283 & 0.0141 & 0.80071 \\
DSR & Rotation & 0.06317 & 4.62941 & 0.75185 & 0.20243 & 12.8656 & 0.0118 & 0.80623 \\
SSR & Average & 0.02489 & 6.99660 & 0.86229 & 0.11733 & 1.62966 & 0.0082 & 0.95034 \\
SSR & Rotation & 0.02589 & 6.92966 & 0.86047 & 0.12060 & 2.86084 & 0.0080 & 0.94248 \\
\hline
\end{tabular}




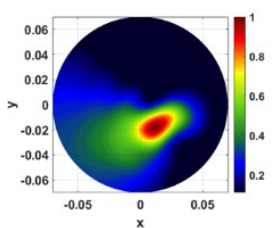

(a)

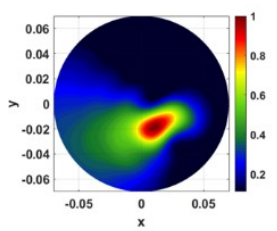

(e)

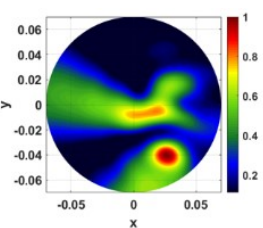

(b)

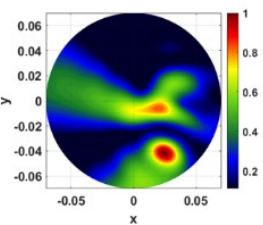

(f)

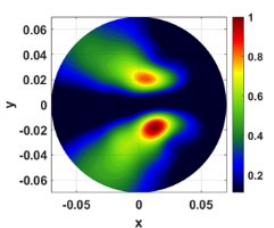

(c)

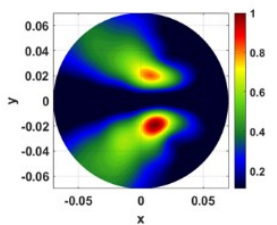

(g)

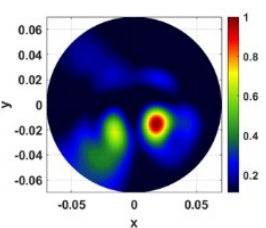

(d)

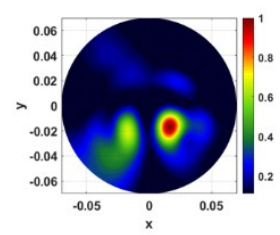

(h)

Fig. 3. Results Images for an Inclusion at $-\frac{\pi}{4}$ radians. Top Row: Average Subtraction - (a) AS, (b) DNR, (c) DSR, (d) SSR. Bottom Row: Rotation Subtraction - (e) RS, (f) DNR, (g) DSR, (h) SSR.

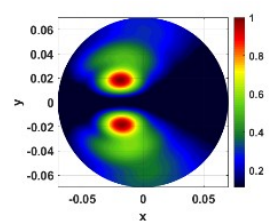

(a)

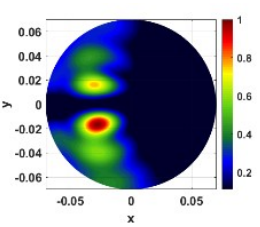

(b)

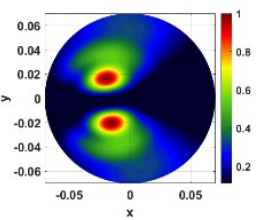

(c)

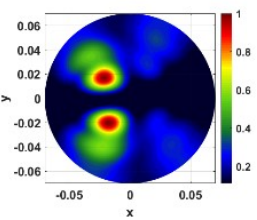

(d)

Fig. 4. Average Subtraction results for an inclusion at $\pi$ radians: (a) AS, (b) DNR, (c) DSR, (d) SSR.

\section{Discussion and Conclusion}

Summed Symmetric Differential method had the best Image Quality Index. The Signalto-Noise Ratio was the highest for this method in both positions and the contrast similarity meant that this method also had a high SSIM in comparison to the other methods.

For an inclusion at $\pi$ radians the Average Subtraction, Rotation Subtraction and Differential (Symmetric Receiver Type) methods all scored highly in the Centroid Distance metric. When the inclusion was at $-\frac{\pi}{4}$ radians, the Summed Symmetric method had the best Centroid Distance score.

The results show that the position of the inclusion can vastly influence the resulting image, with every artefact removal method exhibiting differences in image quality when the inclusion was moved. When the inclusion is at $\pi$ radians, all images show a symmetric split inclusion image. This is likely due to the inclusion being directly in front of the transmitting source. As the image is split perfectly either side of the 
inclusion position, the centroid distance (and to a lesser extent the Area Difference) remain accurate. The SSIM is greatly affected though due to the difference in inclusion shape between the test image and ideal image. In simulation, the source signal will hit the inclusion and the highest peaks are observed as the signal reflections are 'split' by the inclusion. With Average Subtraction and Summed Symmetric Differential methods, there is far greater definition of the inclusion position. To avoid the inclusion position having an impact on the result, multiple transmitter groups could be used. If five transmitter triplets are used, such as in [4] with each transmitter within a triplet placed $4.5^{\circ}$ apart and the triplets placed equally around the perimeter of the cylinder to be imaged, the inclusion will only ever be opposite a maximum of one transmitter triplet. If the results for each triplet are summed, or a mean value taken, and then imaged the resulting image should have improved the definition of the inclusion image, reflected artefacts will be minimised and the dependency on inclusion position will be removed. However, as the Summed Symmetric Differential methods had reflected artefacts which were separate from the inclusion image, the effect of summing the results from multiple transmitters could increase the quantity of artefacts seen for this method. Concerning computational time, we found no significant difference among the algorithms used.

Whilst the Summed Symmetric method scored highly in this study, the experiments were performed using simulated cylinders with significantly contrasting electrical properties. This was performed to prove the algorithms as a proof of concept against contrasting mediums. In a realistic scenario, such as brain stroke detection, the contrast will be less significant, thus artifacts could be more prominent, lowering SNR values. Future studies should aim to use simulated data which equates the electrical properties closer to that of human tissues. Further study using measured data and head-mimicking phantoms would be an obvious progression from this study to assess the value in these methods.

It is also important to note that this study has also focused only on cylindrical inclusions inside a cylinder. Further studies could explore the effect of changing the shape and size of the inclusion.

The Image Quality Index provides a good metric for quantifying the quality of images. By visually comparing the results images and the IQI, the score seems representative of the quality of the image. For a more rigorous assessment of the IQI method, future study could incorporate a comparison with subjective scoring by a sample of medical professionals.

Acknowledgments. This project leading to this application has received funding from the European Union's Horizon 2020 research and innovation programme under the Marie Sklodowska-Curie grant agreement No. 793449.

This project has received funding from the European Union's Horizon 2020 research and innovation programme under the Marie Sklodowska-Curie grant agreement No. 872752 . 


\section{References}

1. Taylor, W., Shah, S.A., Dashtipour, K., Zahid, A., Abbasi, Q.H., Imran, M.A.: An Intelligent Non-Invasive Real-Time Human Activity Recognition System for Next-Generation Healthcare. Sensors 20(9), p.2653 (2020).

2. Klemm, M., Leendertz, A., Gibbins, D., Craddock, I. J., Preece, A., Benjamin, R.: Microwave Radar-Based Differential Breast Cancer Imaging: Imaging in Homogenous Breast Phantoms and Low Contrast Scenarios. IEEE Transactions on Antennas and Propagation 58(7), pp. 2337-2344 (2010).

3. Ghavami, M. Vispa, A., Sani, L., Paoli, M., Bigotti, A., Raspa, G., Ghavami, N., Tiberi, G.: UWB Device for Microwave Imaging: Validation through Phantoms. In: 13th European Conference on Antennas and Propagation, Krakow, Poland (2019).

4. Vispa, A., Sani, L., Paoli, M., Bigotti, A., Raspa, G., Ghavami, N., Duranti, M., Tiberi, G.: UWB device for breast microwave imaging: phantom and clinical validations. Measurement. 146, pp582-589 (2019).

5. Sohani, B., Khalesi, B., Ghavami, N., Ghavami, M., Dudley, S., Rahmani, A., Tiberi, G.: Detection of haemorrhagic stroke in simulation and realistic 3-D human head phantom using microwave imaging. Biomedical Signal Processing and Control. 61 (2020). https://doi.org/10.1016/j.bspc.2020.102001.

6. Elahi, M. A., Glavin, M., Jones, E., O'Halloran, M.: Artifact Removal Algorithms for Microwave Imaging of the Breast. Progress in Electromagnetics Research. 141, pp. 185-200 (2013).

7. Lazebnik, M., Popovic, D., McCartney, L., Watkins, C. B., Lindstrom, M. J., Harter, J. et al.: A large-scale study of the ultrawideband microwave dielectric properties of normal, benign and malignant breast tissues obtained from cancer surgeries. Physics in Medicine \& Biology, 52(20), (2007).

8. Sohani, B., Tiberi, G., Ghavami, N., Ghavami, M., Dudley, S., Rahmani, A.: Microwave imaging for stroke detection: validation on head-mimicking phantom. In: PIERS (Photonics and Electromagnetics Research Symposium), Rome, Italy (2019).

9. Ghavami, N., Tiberi, G., Edwards, D. J., Monorchio, A.: UWB Microwave Imaging of Objects With Canonical Shape. IEEE Transactions on Antennas and Propagation. 60(1), pp. 231-239 (2012).

10. Enders, P.: Huygens' Principle as Universal Model of Propagation. Latin American Journal of Physics Education. 3(1), pp. 19-32 (2009).

11. Parrikar, R. P., Kishk, A. A., Elsherbeni, A. Z.: Scattering from an impedance cylinder embedded in a nonconcentric dielectric cylinder. IEEE Proceedings H: Microwaves, Antennas and Propagation, 138(2), pp. 169-175 (1991).

12. Klemm, M., Craddock, I. J., Leendertz, A., Preece, A., Benjamin, R.: Improved delay-andsum beamforming algorithm for breast cancer detection. International Journal of Antennas and Propagation. Article ID 761402, p. 9 (2008).

13. Mustafa, S., Mohammed, B., Abbosh, A.: Novel Preprocessing Techniques for Accurate Microwave Imaging of Human Brain. IEEE Antennas and Wireless Propagation Letters. 12, pp. 460-463 (2013).

14. Zamani, A., Abbosh, A. M.: Clutter Rejection Techniques for Microwave Head Imaging. In: 2015 International Symposium on Antennas and Propagation (ISAP), Hobart, Australia (2015).

15. MATLAB Documentation Centre, https://uk.mathworks.com/help/documentation-center.html, last accessed 15/05/2020. 
16. Zhou, W., Bovik, A. C., Sheikh, H. R., and Simoncelli, E. P.: Image Quality Assessment: From Error Visibility to Structural Similarity. IEEE Transactions on Image Processing. 13(4), pp. 600-612 (2004). 